\title{
The Filter Detection Task for measurement of breathing-related interoception and metacognition
}

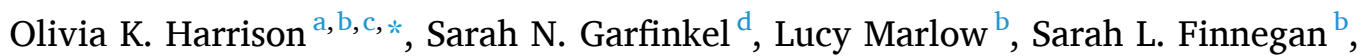 \\ Stephanie Marino ${ }^{\text {a }}$, Laura Köchli ${ }^{\text {a }}$, Micah Allen ${ }^{\text {e,f,g }}$, Johanna Finnemann ${ }^{g}$, \\ Laura Keur-Huizinga $^{g}$, Samuel J. Harrison ${ }^{a}$, Klaas E. Stephan ${ }^{\text {a }}$, Kyle T.S. Pattinson ${ }^{\mathrm{b}}$, \\ Stephen M. Fleming ${ }^{\text {h, }, \mathrm{j}}$
}

\footnotetext{
a Translational Neuromodeling Unit, Institute for Biomedical Engineering, University of Zurich and ETH Zurich, Switzerland

${ }^{\mathrm{b}}$ Nuffield Department of Clinical Neurosciences, University of Oxford, United Kingdom

${ }^{\mathrm{c}}$ Department of Psychology, University of Otago, New Zealand

${ }^{\mathrm{d}}$ Institute of Cognitive Neuroscience, University College London, United Kingdom

${ }^{\mathrm{e}}$ Aarhus Institute of Advanced Studies, Aarhus University, Denmark

${ }^{\mathrm{f}}$ Center of Functionally Integrative Neuroscience, Aarhus University Hospital, Denmark

${ }^{g}$ Cambridge Psychiatry, University of Cambridge, United Kingdom

${ }^{\mathrm{h}}$ Wellcome Centre for Human Neuroimaging, University College London, United Kingdom

${ }^{\mathrm{i}}$ Max Planck Centre for Computational Psychiatry and Ageing Research, University College London, United Kingdom

${ }^{\mathrm{j}}$ Department of Experimental Psychology, University College London, United Kingdom
}

\section{A R T I C L E I N F O}

\section{Keywords:}

Interoception

Breathing

Inspiratory resistance

Metacognition

\begin{abstract}
A B S T R A C T
The study of the brain's processing of sensory inputs from within the body ('interoception') has been gaining rapid popularity in neuroscience, where interoceptive disturbances are thought to exist across a wide range of chronic physiological and psychological conditions. Here we present a task and analysis procedure to quantify specific dimensions of breathing-related interoception, including interoceptive sensitivity, decision bias, metacognitive bias, and metacognitive performance. Two major developments address some of the challenges presented by low trial numbers in interoceptive experiments: (i) a novel adaptive algorithm to maintain task performance at 70-75\% accuracy; (ii) an extended hierarchical metacognitive model to estimate regression parameters linking metacognitive performance to relevant (e.g. clinical) variables. We demonstrate the utility of the task and analysis developments, using both simulated data and three empirical datasets. This methodology represents an important step towards accurately quantifying interoceptive dimensions from a simple experimental procedure that is compatible with clinical settings.
\end{abstract}

\section{Introduction}

Understanding how the brain integrates sensory information to guide perception and action is a core component of neuroscientific research. Whilst the mapping of sensory pathways and perceptual phenomena have seen major developments in our understanding of the 'exteroceptive' domain (such as vision, audition, touch etc.), the study of 'interoception' (or the brain's processing of sensory inputs from within the body) has begun receiving attention only relatively recently (Khalsa et al., 2017). While theoretical concepts of the dynamic interplay of brain and body - including interoception, homeostatic and allostatic control (Allen, 2020; Petzschner, Weber, Gard, \& Stephan, 2017; Pezzulo, Rigoli, \& Friston, 2015; Stephan et al., 2016) - exist, empirical investigations have lagged behind. However, empirical studies of interoception have been recently boosted by a surge of interest in multiple neuroscientific fields, given that impairments in interoceptive processing have been proposed to play a role in emotions, decision making, consciousness and mental health (Khalsa et al., 2017; Owens, Allen, Ondobaka, \& Friston, 2018).

Perceptual processing is a complex phenomenon, and one that is highly integrated with other domains of brain function. For example, visual perception can be manipulated via changes in factors such as

\footnotetext{
* Corresponding author at: Translational Neuromodeling Unit, Institute for Biomedical Engineering, University of Zurich and ETH Zurich, Switzerland.

E-mail address: faull@biomed.ee.ethz.ch (O.K. Harrison).
} 
attention (Brefczynski \& DeYoe, 1999), emotional state (Bocanegra \& Zeelenberg, 2009) or expectation (Summerfield \& Egner, 2009). Furthermore, objective performance in perceptual detection tasks (i.e. accuracy and sensitivity towards stimulus detection, as often measured with classic psychophysics experiments (Kingdom \& Prins, 2016) and using inspiratory loading paradigms (Chou \& Davenport, 2007; Daubenmier, Sze, Kerr, Kemeny, \& Mehling, 2013; Davenport, Chan, Zhang, \& Chou, 2007; Garfinkel et al., 2016a; Ruehland, Rochford, Trinder, Spong, \& O'Donoghue, 2019)) can be differentiated from more 'metacognitive' dimensions, where metacognition refers to the ability to accurately reflect and monitor cognitive or perceptual processes (Fleming \& Lau, 2014; Fleming, 2017; Garfinkel et al., 2016a; Garfinkel, Seth, Barrett, Suzuki, \& Critchley, 2015; Maniscalco \& Lau, 2012). To quantify aspects of metacognition, measures of task performance can be paired with judgements of the confidence assigned to a decision (Fleming \& Lau, 2014; Garfinkel et al., 2015, 2016a, 2016b). From these metrics, average confidence can be thought of as a 'metacognitive bias', or a tendency towards a certain level of confidence, while 'metacognitive performance' (or 'metacognitive sensitivity') reflects how well confidence measures align with actual task performance (Fleming, 2017; Fleming \& Lau, 2014; Garfinkel et al., 2015; Maniscalco \& Lau, 2012). Critically, to distinguish these metacognitive measures from underlying task performance, either objective accuracy needs to be held consistent across participants, or the effect of task accuracy needs to be accounted for using appropriate mathematical models (and an adequate volume of data acquired to fit these models) (Fleming \& Lau, 2014). Here, we propose a method that incorporates both control over task performance, as well as accounting for any residual task performance variation between individuals.

These dimensions - task performance, metacognitive bias and metacognitive performance - have been distinguished within an interoceptive model by Garfinkel and colleagues (Garfinkel et al., 2015). Here, the authors demonstrated that these domains appear to be both quantifiable and distinct, and potentially related to traits such as anxiety. While their study was focused on cardiac-related body signals, initial work has hinted at potential cross-talk across different interoceptive 'channels' in the metacognitive domain, where corresponding interoceptive metacognition (but not task performance) was observed across cardiac and respiratory tasks (Garfinkel et al., 2016a). Interestingly, this study also reported significantly elevated confidence in breathing-related perceptual decisions when compared to judgements of cardiac and tactile performance (Garfinkel et al., 2016a). Whilst breathing is more consciously accessible for both perception and control than the cardiac domain, this elevated confidence also highlights the importance and relevance of breathing-related symptoms in the maintenance of homeostasis, whereby even a single breath of restricted or occluded breathing can be perceived as extremely unpleasant and frightening (Paulus, 2013).

Central for the further development of interoceptive research is the requirement to develop robust methodologies that can quantify interoceptive and metacognitive dimensions. Breathing is often considered to lie at the border of interoception and exteroception, combining cues from sensory avenues such as tactile and skeleto-muscular sensations across the chest wall, muscular effort, blood-gas signals representing bodily respiratory status, and air temperature and humidity, to name a few. Importantly, the accessibility of breathing to voluntary alterations and conscious perceptions lends itself to an array of experimental paradigms, including those that do not require exteroceptive cues. In a similar manner to cardiac measures, breathing contains inherent variability in flow and resistance both between and within individuals. These individual and breath-by-breath differences render highly accurate measures of breathing-related perceptual sensitivity challenging. However, if the performance of a perception task is both controlled and accounted for, metacognitive measures relating to interoception can become both accessible and independent of these challenges.

In this paper we provide a novel methodology for controlling task performance on a breathing perception task, and demonstrate the utility of applying a computational modelling approach to analyze metacognitive metrics of breathing perception. The main benefit of utilizing this computational model to assess metacognitive performance is that the effect of underlying task performance on metacognition can be removed (Fleming, 2017; Maniscalco \& Lau, 2012), as differences in task performance will produce concurrent differences in apparent metacognition if not adequately accounted for (Fleming \& Lau, 2014). Importantly, an experimental setup is employed that is sufficiently simple and mobile to enable practical applications outside a laboratory setting, providing progress towards more useful clinical assessments of interoceptive properties of breathing. Inspiratory resistance is used as the breathing stimulus in this task, as it is both controllable and relevant to many individuals; for example, changes in airway resistance and pressure can result from both bronchoconstriction in conditions such as asthma and/or panic disorder (Smoller, Pollack, Otto, Rosenbaum, \& Kradin, 1996). However, inspiratory pressure also changes in physiological conditions, for example, simply as a result of increased inspiratory flow during activities such as exercise (Johnson, Weisman, Zeballos, \& Beck, 1999) or hyperventilation induced by states of arousal (Gallego, Nsegbe, \& Durand, 2001), but also as the result of reflex-mediated bronchoconstriction in response to cooling of the skin or upper airways (Koskela, 2007; Koskela \& Tukiainen, 1995). Furthermore, it is now widely acknowledged that the perceptual system can be influenced by top-down factors such as attention, expectation and affect (Bogaerts et al., 2005, 2008; Van den Bergh, Witthöft, Petersen, \& Brown, 2017; Janssens, Verleden, De Peuter, Van Diest, \& Van den Bergh, 2009, 2011; Marlow, Faull, Finnegan, \& Pattinson, 2019; De Peuter et al., 2004; De Peuter, Lemaigre, Van Diest, \& Van den Bergh, 2008; Put et al., 2004; Stephan et al., 2016; Van den Bergh et al., 2004); a known issue in conditions where symptoms are discordant with objectively measured medical markers, such as in asthma (Boulay \& Boulet, 2013; Janssens et al., 2009; Kendrick, Higgs, Whitfield, \& Laszlo, 1993; Teeter \& Bleecker, 1998) or those with medically unexplained symptoms (Isaac \& Paauw, 2014; Nimnuan, Hotopf, \& Wessely, 2001; Steinbrecher, Koerber, Frieser, \& Hiller, 2011). Therefore, using a task that is able to dissociate measures such as perceptual sensitivity from decision bias and metacognition has great potential to fill an important unmet need in clinical practice.

To firstly demonstrate the utility of computational modelling for breathing, we combined an interoceptive breathing task based on resistive loads (Garfinkel et al., 2016a) with an established computational model of metacognition (HMeta-d) (Fleming \& Lau, 2014; Fleming, 2017; Maniscalco \& Lau, 2012). We utilized both simulations and empirical data from individuals with asthma, as a cohort of individuals who regularly experience changes in airway resistance. The HMeta-d model utilizes a robust hierarchical statistical framework that allows computational models of metacognition, such as the meta-d model (Maniscalco \& Lau, 2012), to be fit to task data where only low numbers of trials are available, such as those from interoceptive tasks. Furthermore, we present an extension to a hierarchical Bayesian model of metacognitive efficiency, HMeta-d (Fleming, 2017), that allows measures of metacognitive performance to be directly regressed against external variables of interest within the hierarchical model. This is an advantage over standard approaches as it capitalizes on the power of hierarchical estimation, especially when trial numbers are low, but avoids the problems encountered by post-hoc regressions on hierarchical model parameters such as unwanted shrinkage to the group mean. This shrinkage within standard hierarchical models is where individual subject estimates are drawn towards the group mean, and the variance between subjects (and thus the ability for post-hoc regression models to accurately explain inter-individual variance) is reduced. Lastly, we present an adaptive task-performance algorithm that directly targets a perceptual threshold accuracy of $\sim 70 \%$ and allows online control of performance, to aid the collection of a maximal number of trials at this level of task difficulty. Importantly, quantification of higher-order 
metacognitive measures is most efficient when objective performance is both significantly above chance (or guessing: $50 \%$ ) and lower than a ceiling value of $100 \%$, such that the resulting perceptual errors can be used to quantify the correspondence between objective accuracy and subjective confidence reports (Fleming \& Lau, 2014). The utility of the analysis models and task-performance algorithm are established using both simulations and empirical data.

\section{Methods}

The Methods section firstly contains an overview of how the Filter Detection Task (FDT) is carried out, followed by an explanation of the four interoceptive measures that can be quantified. We then describe the computational model simulations employed to determine the applicability of these analysis methods to limited-trial interoceptive applications. Next, we describe the testing and analysis methods employed to assess illustrative hypotheses in an example empirical dataset that encompassed a group of individuals with asthma as well as healthy controls. Finally, we describe the novel task algorithm that was designed to both control performance (within sessions and between individuals) and increase the number of trials measured at the perceptual threshold of an individual, which is defined using the classical value of $70-75 \%$ (Watson \& Pelli, 1983), lying half-way between guessing between the two answer options available (50\%) and a ceiling value of $100 \%$. We use both simulations and two further empirical datasets to demonstrate the utility of the algorithm to control performance and reduce the number of excess (unused) trials.

\subsection{Filter Detection Task overview}

To systematically test breathing perception within interoception, we have developed a perceptual threshold breathing task (the FDT) based on a previously-reported perceptual breathing task (Garfinkel et al., 2016a). This task is a perceptual discrimination task, and can either be completed as a 'Yes/No' decision task, or a two-interval forced choice (2IFC) task. This task requires a computer to run MATLAB, as well as low-cost and easily accessible spirometry filters and anesthetic tubing, presenting a simple setup (Fig. 1) that allows for assessment of multiple interoceptive and metacognitive measures within the breathing domain. Participants wear a nose clip throughout the task, such that breathing is performed only through the mouth.

For the 'Yes/No' version of the task, a standard trial structure consists of participants first taking three 'baseline' breaths through a mouthpiece connected to a simple breathing system (outlined in Fig. 1). Following the baseline breaths, three breaths take place under 'resistance' or 'sham' conditions: either an inspiratory load is created via the addition of combinations of clinical breathing filters (signal trials, filters provided by GVS Filter Technology, product number 2800/22BAUF), or an empty filter (sham trials) is added to the system. The filters provide a resistance of $<0.48 \mathrm{~cm} \mathrm{H}_{2} \mathrm{O} / \mathrm{L} \cdot \mathrm{sec}^{-1}$ with the filter membrane attached (see Supplementary Material for further information). After each trial, participants are asked to verbalize their decision as to whether or not a load had been added. Alternatively, for the 2IFC task, the inspiratory load is either added in the first or second set of three breaths, and participants are asked to choose in which of the two intervals the inspiratory load was present. In either task, after each trial participants are asked to verbally report their confidence in this decision on a userdefined scale, e.g. from 1 to $10(1=$ not at all confident in decision, $10=$ extremely confident in decision). The use of verbal feedback has the additional advantage of momentarily taking the participant's attention away from their breathing. Participants can also take any length of rest period required between each trial.

The responses from the FDT can then be used to determine a variety of interoceptive measures (outlined below), including inspiratory loadrelated perceptual sensitivity, bias in symptom reporting, perceptual confidence and metacognition (the ability to accurately reflect upon cognitive or perceptual processes).

\subsection{Breathing-related interoceptive measures}

In the original version of the filter detection task (Garfinkel et al., 2016a), three domains of breathing-related interoception were quantified: interoceptive sensitivity (number of filters required for a discrimination threshold of approximately 75\%), interoceptive sensibility (average confidence over threshold trials) and metacognition (correspondence between accuracy and confidence using a Type 2 receiver
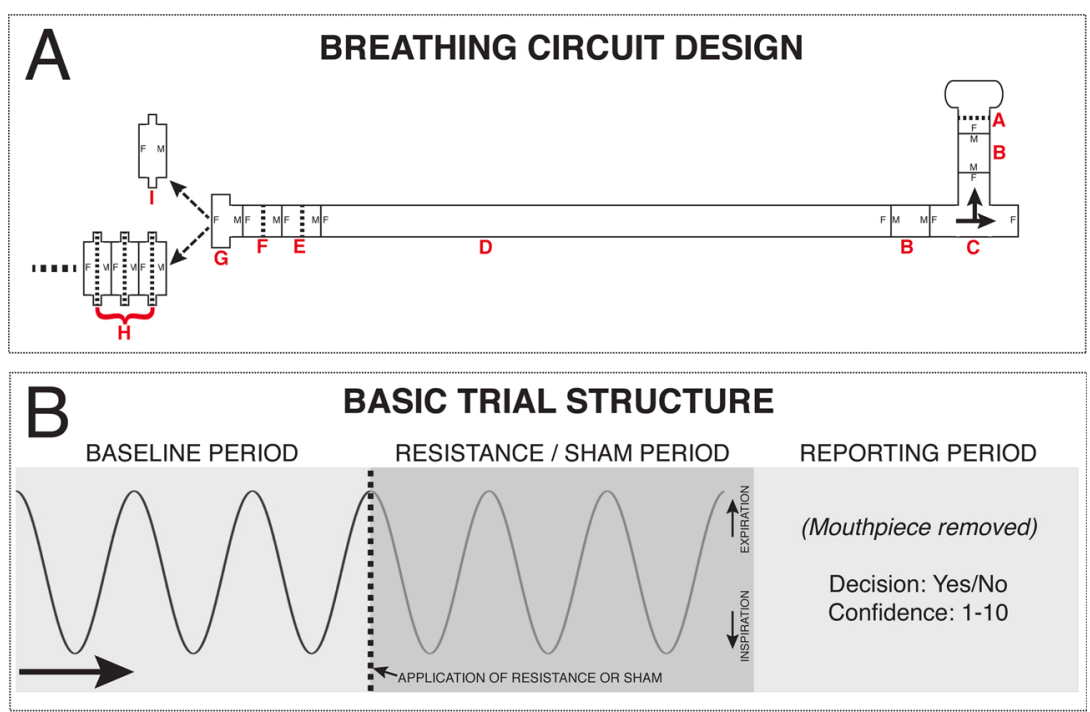

Fig. 1. A) Diagram of circuitry for the filter detection task. A single-use, bacterial and viral mouthpiece (A: Powerbreathe International Ltd., Warwickshire, UK - Product SKU PBF03) is attached to a $22 \mathrm{~mm}$ diameter connector (B: Intersurgical Ltd., Berkshire, UK - Product 1960000) and a t-shaped inspiratory valve (C: Hans Rudolf, Kansas City, MO, USA - Product 1410/ 112622), connected to a $2 \mathrm{~m}$ length of $22 \mathrm{~mm}$ diameter flexible tubing (D: Intersurgical Ltd. - Product 1573000) and two additional baseline filters (E: Intersurgical Ltd. - Product 1541000, and F: GVS, Lancashire, UK - Product 4222/ 03BAUA). A 22-30 mm (G: Intersugical Ltd. - Product 197100) adapter then allows the attachment of either a series of connected spirometry filters (H: GVS - Product 2800/17BAUF, Pressure at $30 \mathrm{~L} / \mathrm{min}<0.3 \mathrm{~cm} \mathrm{H}_{2} \mathrm{O}$, Resistance $<0.48 \mathrm{~cm}$ $\mathrm{H}_{2} \mathrm{O} / \mathrm{L} \cdot \mathrm{sec}^{-1}$ ) or a sham 'dummy' filter - a spirometry filter shell with the inner bacterial protection pad removed (I). B) Overview of the basic trial structure for a Yes/No formulation of the task. Participants take three normal size/pace breaths (with the sham filter attached), and during the third exhalation (indicated by the participant raising their hand and the dotted line in panel B) the experimenter either swaps the sham for a number of stacked filters (to provide a very small inspiratory resistance) or removes and replaces the sham filter. Following three more breaths, the participant removes the mouthpiece and reports whether they thought it a resistance was added ('Yes') or not ('No'), and how confident they are in their decision on any scale (here 1-10 used, with $1=$ guessing and $10=$ maximally confident in their decision). If a two-interval forced choice (2IFC) formulation of the task is used, the filters (resistance) are either placed on the circuit for the first three breaths or the second three breaths according to the FDT algorithm, with the sham filter on the system during the alternate period. The reported decision from the participant is whether they thought the resistance was on in either the first set or the second set of three breaths, and also again the confidence in their decision. 
operating characteristic curve; an ROC curve). Here, we aimed to extend these measures to incorporate a more thorough and nuanced overview of a range of interoceptive dimensions. These measures include interoceptive sensitivity, decision bias, metacognitive bias, and metacognitive performance.

\subsubsection{Interoceptive sensitivity}

This measure is analogous to interoceptive accuracy, and in this task aims to quantify the 'perceptual threshold' as a means for determining breathing-related interoceptive sensitivity. In other words, interoceptive sensitivity here is the level (i.e. number of filters) at which a participant is able to consciously detect an inspiratory loading stimulus. However, to allow for the quantification of higher-order interoceptive measures (such as metacognition), the number of filters used must elicit a performance that is both significantly above chance (or guessing) and lower than $100 \%$, so that perceptual errors can be used to quantify the correspondence between accuracy and confidence (as explained below in 'metacognitive performance'). To achieve a task difficulty that elicits this required performance, the original publication of this task (Garfinkel et al., 2016a) utilized a descending accuracy staircase protocol, whereby a large starting filter number (for example 7 filters), and 20 trials were completed at descending filters until a final filter level when performance first dropped below $70 \%$. While many staircase protocol options could be adopted to achieve a desired task difficulty, the time to complete one trial (approximately 30-60 seconds), the natural variability in resting tidal breaths (Khatib, Oku, \& Bruce, 1991), the inherent bias associated with the Yes/No task formulation (Peters, Ro, \& Lau, 2016) and the fixed filter intervals render many traditionally-employed staircase protocols (such as the two/three-down-one-up (Kingdom \& Prins, 2016)) less straightforward with the current methodology. Therefore, we have developed a custom staircase algorithm (explained in 'Task performance algorithm') that employs probability metrics to objectively assess both task performance and trajectory towards the required perceptual threshold (see 'Task performance algorithm' section for full explanation of the algorithm).

\subsubsection{Interoceptive decision bias}

If using the FDT as a Yes/No task, a quantifiable measure of behavior is the 'bias' towards reporting 'Yes' or 'No'. This bias represents the placement of a criterion value above which the presence of a resistance is reported, reflecting an individual's inherent tendency to report the presence of an inspiratory resistance. Importantly, this bias can be quantified using Signal Detection Theory (SDT (Green \& Swets, 1966; Stanislaw \& Todorov, 1999)), and may represent an important cognitive trait regarding the experience of respiratory symptoms. Using SDT, stimulus sensitivity (d') can be separated from bias (or the placement of a criterion, c) (Green \& Swets, 1966; Stanislaw \& Todorov, 1999). As such, we are able to disentangle the components of measures that may be confounded by a mix of task sensitivity and bias, such as performance accuracy. When using the FDT as a 2IFC task, the measured 'bias' will instead be the tendency to report the resistance on the first or second interval. While this may have limited relevance to real world scenarios, quantification of d' using this task design is likely to also allow for a more accurate representation of task sensitivity and a possibly more translatable measure of metacognitive performance (Lee, Ruby, Giles, \& Lau, 2018), which can both be confounded by variations in criterion placement.

\subsubsection{Interoceptive metacognitive bias}

Average subjective confidence or metacognitive bias in interoceptive decisions has also previously been referred to as interoceptive "sensibility" (Garfinkel et al., 2015). In this task, we take an overall average of the confidence scores (measured across the perceptual threshold trials) to represent interoceptive sensibility that directly corresponds to the task at hand. Additional trait-like, global perceptual measures of sensibility could also be gathered by using separate interoceptive questionnaires such as the Porges Body Questionnaire (Porges, 1993). Interoceptive sensibility is also referred to as "metacognitive bias" (Fleming \& Lau, 2014; Fleming, 2017; Rouault, Seow, Gillan, \& Fleming, 2018), as it represents the tendency to give higher or lower confidence ratings.

\subsubsection{Interoceptive metacognitive performance}

Breathing-related metacognitive performance (also termed 'interoceptive awareness' (Garfinkel et al., 2015) and 'interoceptive insight' (Khalsa et al., 2017) in the literature) in this instance is considered to be the correspondence between task accuracy and confidence (Garfinkel et al., 2015), or the ability to recognize successful perceptual processing (Fleming \& Lau, 2014). Previous reports of interoceptive metacognition have utilized the area under a type 2 ROC curve (Garfinkel et al., 2015, 2016a, 2016b), resulting in a measure of absolute metacognition where the effect of underlying task performance also influences the final score (Fleming \& Lau, 2014). However, more recent model-based approaches have developed a metric of metacognitive performance that can subsequently take into account task performance, known as meta-d' (Maniscalco \& Lau, 2012). Meta-d' represents "the sensory evidence available for metacognition in signal-to-noise ratio units" (Fleming \& Lau, 2014), which, because it is in the same units as d', can be straightforwardly compared to task performance as a ratio (Mratio: meta-d'/d'; the log of the ratio, logMratio, is also often used to meet Gaussian assumptions: log (meta-d'/d')). This ratio of absolute metacognitive performance (meta-d') divided by task performance (d') is thus a relative measure of metacognitive performance (often termed 'metacognitive efficiency'). In order to employ this model-based approach for the FDT, a hierarchical formulation of the meta-d model (HMeta-d) is employed that allows efficient pooling of data from multiple subjects (Fleming, 2017), allowing us to estimate model parameters on a relatively small number of threshold trials ( $\geq 40$ trials).

\subsection{Metacognitive model simulations}

To demonstrate the feasibility of utilizing the meta-d model for interoceptive tasks with low trial numbers, we first present simulated results to establish the recoverability of group metacognitive performance (Mratio) values using the original maximum likelihood estimation algorithm (MLE (Maniscalco \& Lau, 2012)), a single-subject Bayesian model and a hierarchical (group) Bayesian model (HMeta-d), which are both provided in the HMeta-d toolbox (Fleming, 2017). Parameter inference in the HMeta-d toolbox rests on a Markov chain Monte Carlo (MCMC) sampling procedure, implemented using the JAGS software package (http://mcmc-jags.sourceforge.net). The extent of the group Mratio recoverability is demonstrated for 20, 40 and 60 trials per subject, with 30 simulated participants. Simulations were generated from a set of values $N(\mu, \sigma)$, which refer to Gaussians parameterized by a mean and standard deviation, using the metad_sim function provided in the HMeta-d toolbox (Fleming, 2017). The meta-d' values for the first set of simulations were generated from seven group Mratio distributions

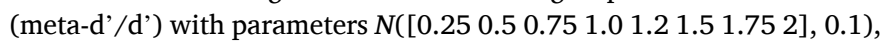
where d' $\sim N(1,0.1)$ and $\mathrm{c} \sim N(0,0.1)$.

Second, we developed and simulated an extension of the HMetad model (RHMeta-d), which incorporates a simultaneous hierarchical estimation of a regression parameter (beta) that controls variation in logMratio values in relation to a subject-level predictor (such as a clinical score). The model was adjusted as follows (for full details of the original model please see the original publication (Fleming, 2017)). $N(\mu$, $\sigma)$ and $H N(\mu, \sigma)$ refer to Gaussians and Half-Gaussians parameterised by a mean and standard deviation, while $T(\mu, \sigma, \nu)$ refers to a T-distribution parameterised by a mean, standard deviation and degrees of freedom: 


$$
\begin{gathered}
M_{0} \sim N(0,1) \\
\beta \sim N(0,1) \\
\sigma_{\delta} \sim H N(1) \\
\zeta \sim \operatorname{Beta}(1,1) \\
\delta_{s} \sim T\left(0, \sigma_{\delta}, 5\right) \\
\varepsilon_{s}=\zeta * \delta_{s} \\
M_{s}=M_{0}+\beta * X_{s}+\varepsilon_{s}
\end{gathered}
$$

where $M_{s}$ refers to the $\log \left(\right.$ meta-d'/d') value for subject $s, M_{0}$ is the baseline logMratio for the group (i.e. the intercept of the regression), and $X_{s}$ is a vector of predictor values (e.g. clinical scores) for each subject. This formulation embeds the estimation of psychopathologycognition relationships into the parameter inference routine, such that the group-level posterior over $\beta$ reflects the influence of individual differences in $X$ on metacognitive performance (Moutoussis, Hopkins, \& Dolan, 2018). To ensure that the regression is robust to outliers, the noise $\varepsilon_{s}$ is drawn from a T-distribution with a standard deviation of $|\zeta| \sigma_{\delta}$ and 5 degrees of freedom (Gelman et al., 2020). Consistent with the original HMeta-d model (Fleming, 2017), a redundant multiplicative parameter $\zeta$ is used to introduce an additional random component in the sampling process to aid the recovery of the posterior on the noise scale.

For these simulations, group baseline $\log$ Mratio $\left(M_{0}\right)$ values were generated from a distribution $\sim N(\log (0.8), 0.1)$. Then, to simulate data from each subject, a random value of the covariate $X_{s}$ was drawn from a standardised distribution $N(0,1)$, multiplied by a group regression coefficient that was one of a fixed set of $\beta$ values ( $\beta \sim$ [-0.5:0.5]) and added to this baseline $\log$ Mratio together with zero-mean noise sampled from $N(0,0.1)$. Values used for d' and c' were consistent with previous simulations ( $\mathrm{d}^{\prime} \sim N(1,0.1)$ and $\mathrm{c} \sim N(0,0.1)$ ), and data were generated using either 20,40 or 60 trials per subject and a confidence scale of 10 rating points. From these simulated data, parameter estimates were then obtained either using the original HMeta-d model combined with a postfit linear regression (i.e. a standard linear regression conducted on the per-subject point estimates obtained from the group-level fit, which we denote HMeta-d $+\mathrm{R}$ ), as well as the extended hierarchical regression model (RHMeta-d) described above. An average for ten sets of simulations for each group $\beta$ value was calculated for the final results.

\subsection{Analysis methods: Example dataset of asthma and healthy controls}

To demonstrate the utility of the analysis methods, we employed an example dataset that included a group of individuals who experience an elevated frequency of breathing symptoms. While it has been observed that individuals with asthma can vary from under-reporting to overreporting of symptoms (Boulay \& Boulet, 2013; Janssens et al., 2009; Kendrick et al., 1993; Teeter \& Bleecker, 1998), group-wise analyses of asthma have demonstrated both an elevated prevalence of anxiety and depression symptoms (Cooper et al., 2007) and that symptom prevalence is related to these affective qualities (Katon, Richardson, Lozano, \& McCauley, 2004; De Peuter et al., 2008; Richardson et al., 2006; Rimington, Davies, Lowe, \& Pearson, 2001). Furthermore, as the metacognitive properties of individuals with asthma have not yet been systematically tested and could viably relate to symptom reporting, this group was selected as an example test-case for this method. Importantly, the FDT allows us to separate the effect of interoceptive sensitivity to inspiratory loads, bias towards under- or over-reporting the presence of a resistance, metacognitive bias (higher or lower confidence in interoceptive decisions) and metacognitive performance ('insight' into perceptual performance). These separable entities may help to shed light on the potential drivers towards the under- to over-reporting of symptoms (Boulay \& Boulet, 2013; Janssens et al., 2009; Kendrick et al., 1993; Teeter \& Bleecker, 1998) in asthma.

Sixty-three individuals with asthma (39 females, mean age ( \pm sd) $43.7 \pm 12.2$ years, recruited through general practitioner clinics and public advertisements) and 30 healthy controls (19 females, mean age ( \pm sd) $44.2 \pm 12.2$ years, recruited through public advertisements) took part in a study approved by the Oxfordshire Clinical Research Ethics Committee. Participants underwent the FDT and completed the Dyspnea 12 (Yorke, Moosavi, Shuldham, \& Jones, 2010) questionnaire as a subjective assessment of their breathlessness severity. Additionally, participants completed a further set of questionnaires and additional physiological and behavioural measures that will be addressed elsewhere. Seven individuals with asthma were excluded from the analysis due to insufficient data (10 trials or less of the FDT, $n=4$ ), or performance of less than $50 \%$ correct $(n=3)$, as determined in the preregistered analysis plan (https://gitlab.ethz.ch/tnu/analysis-plans/h arrisonetal_fdt_methods_2020).

During the FDT in this study, the number of filters required for each participant to induce task performance at perceptual threshold was determined manually, using a staircase method adjusted from a previous publication of the task (Garfinkel et al., 2016a) (data was collected prior to the development of the task algorithm). In this step-wise procedure, participants first completed 10 trials at 4 filters. If the task accuracy was below or above $70 \%$, the number of filters was adjusted up or down accordingly by one filter. Performance accuracy was assessed again at 20,30 and 40 trials, with adjustments made if the accuracy moved outside of the $65-75 \%$ range (with an acceptable range of $60-80 \%$ in later trials). The aim was to complete $40-60$ trials, which was limited by time and attention constraints of each participant. A 0-100 confidence rating scale was employed, with $0=$ not at all confident, and $100=$ maximal confidence. These confidence scores were down-sampled into 10 rating bins prior to analysis with the HMeta-d model.

To demonstrate empirical questions that could be answered using the FDT and metacognitive models, we firstly tested any differences between individuals with asthma and healthy controls across all FDT parameters (https://gitlab.ethz.ch/tnu/analysis-plans/harrisonetal_fdt _methods_2020). For the measures of interoceptive sensitivity (number of filters), decision bias (c parameter from model) and metacognitive bias (average confidence), tests for data normality were first conducted using Anderson-Darling tests, with an alpha value of $p<0.05$ required to reject the null hypothesis of normally distributed data. A significant group difference was then tested using two-tailed parametric or nonparametric Wilcoxon rank-sum tests. A group difference in metacognitive performance (the Mratio parameter from the HMeta-d model output) was assessed by first calculating the distribution of differences in posterior parameter samples from each group (control Mratio samples > asthma Mratio samples), and then determining the highest density interval (HDI) for this distribution. The HDI employed was a two-tailed 99\% (Bonferroni corrected for five tests, including the regression analysis described below) confidence interval, where a significant difference between groups was denoted if the resulting HDI did not span zero. Significance for all other tests was denoted by $\mathrm{p}<0.01$ ( $\mathrm{p}<0.05$ Bonferroni corrected for the five experimental tests).

To assess a possible relationship between metacognitive performance and the prevalence of reported breathing-related symptoms, a direct test of the relationship between metacognitive performance and D12 was performed using a linear regression model in the individuals with asthma. To achieve this, the HMeta-d model was extended to include a hierarchical estimation of a linear regression parameter, whereby a group regression coefficient (beta) was simultaneously fit within the model to determine the relationship between logMratio and D12. The significance of the beta parameter was then also determined using its posterior samples, with a two-tailed 99\% HDI (Bonferroni corrected) that did not span zero determining a significant relationship between more severe D12 and worsened metacognitive performance. An illustrative procedure using a split-half analysis is presented in the Supplementary Material.

\subsection{Task performance algorithm: Simulations and empirical data}

Lastly, to assist in the collection of a greater number of usable trials for further instances of the FDT, we created a novel staircase protocol 
(within a MATLAB toolbox package) to aid the selection of the appropriate number of filters for each participant. While adaptive psychophysics staircase algorithms are available (such as QUEST (Watson, 2017; Watson \& Pelli, 1983)), many of these formulations rely on adjustable step-sizes and small amounts of sensory noise, and also often assume a pre-determined psychometric for model fitting. Therefore, for this novel application in the breathing domain we designed an algorithm that does not assume any psychometric function; instead, it estimates the underlying accuracy that gives rise to the current performance using a beta-binomial model. This simple model is robust and does not run the risk of non-convergence as can be observed with a more complicated algorithm such as QUEST, which may occur due to both the limited number of trials, step sizes available and variability in breathing from trial to trial.

When interfacing with the task algorithm, the researcher is given instructions for each trial via the MATLAB command window. Additionally, the researcher is required to enter the participant's decision and confidence scores into the MATLAB command window when prompted for every trial, and this information is then used to dynamically update the staircase procedure. This staircase begins with one or two practice trials and a short calibration, which are completed under the same within-trial format (i.e. Yes/No or 2IFC structure) as the main task trials. In the practice trials for the Yes/No task, an additional 'explicit dummy' is first applied, whereby participants are told that it is a dummy. Another practice trial is then performed using a large load of 7 filters, where no feedback is given (no feedback is maintained for the rest of the protocol). The practice is then immediately followed by the calibration trials, where participants complete trials that increase by one filter each trial (beginning with a dummy) until they have correctly reported the resistance for two consecutively increasing filter numbers. A final calibration trial is then given, where the number of filters is dropped by one from the last trial. If participants correctly report the final calibration trial, they begin on that number of filters, whereas if they are incorrect, they begin with one additional filter. A diagram of the basic trial structure is presented in Fig. 1, and the practice, calibration and real trial trajectory is provided in the Supplementary Material (Supplementary Fig. 1).

Once the calibration is complete (or alternatively, a manual starting point can be provided), the main task trials begin. The target number of trials is specified (recommendation of $\geq 60$ trials), and a pseudorandomized sequence of trials are presented (trials are balanced between present/absent for a Yes/No task or between first and second interval for 2IFC). The target is for participants to be within a $65-80 \%$ accuracy band. Given a set of binary trials (and an appropriate prior), we use the fact that the posterior distribution over the underlying accuracy follows a beta distribution. After 5 trials at one filter level (with at least one resistance present for the Yes/No task), the posterior probability that the underlying performance accuracy for the current task difficulty (i.e. the current number of filters) falls between $65-80 \%$ is calculated using the difference in beta cumulative distribution functions for $80 \%$ and $65 \%$, in a similar vein to the QUEST algorithm (Watson \& Pelli, 1983). We use a weak prior on the accuracy itself (beta distribution with the parameters $\alpha=2$ and $\beta=1$; prior mean $=67 \%$ accuracy, interquartile range $=37 \%$ ), determined by the performance of simulations to produce observed accuracies closest to $75 \%$. If the probability that the underlying accuracy is between $65-80 \%$ falls below a threshold of $20 \%$, an addition or removal of a filter is automatically suggested to decrease or increase task difficulty respectively. If a new filter number is started, the trial count will begin again (i.e., a 'constant' staircase design) and 5 trials (with at least one resistance) must be completed before the algorithm will suggest any changes. If the filter change moves the filter number back to that of previous trials, the trial count will pick up again from the last trial at this level. In this instance, 3 trials (with at least one resistance) must be completed before any changes are suggested. If a 'roving' staircase design is specified, trial count will continue regardless of filter number. In this scenario, the 'threshold' filter becomes the average of the number of filters employed across the task.

To demonstrate the utility of this task performance algorithm, we firstly present simulation results from a range of possible participant performances, characterized by a distribution of potential psychometric functions. These psychometric functions $(n=350)$ were constructed from an underlying logistic sigmoid with a lower asymptote at 0.5 (to account for chance answers with the two answer options of 'yes' and 'no'), a slope $k=[0.7: 1.2]$, the number of filters at which the $75 \%$ threshold is obtained $t=[1: 7]$ and added Gaussian noise $\varepsilon=N(0$, [0.05:0.015]). We then ran each of the sigmoids generated from each of 5 starting points - from two filters below to two filters above the $t$ parameter, totaling 7000 simulations. Second, we provide data metrics (number of trials, performance accuracy, number of filters) for two collected datasets using the Yes/No version of the task. The first of these collected datasets stems from the first 50 participants measured as part of a wider study approved by the Cantonal Ethics Committee Zurich (Ethics approval BASEC-No. 2017-02330). For this study, we employed a 'constant' staircase formulation of the algorithm, with the aim of collecting 60 trials at a single level of filters that elicited a task performance between $\sim 60-85 \%$. The second empirical dataset includes the first 22 participants measured as part of a wider study approved by the Cambridge ethics committee (Ethics approval PRE.2018.092). This study employed the alternative 'roving' staircase with 60 trials total, where the aim was to simply collect 60 trials regardless of the number of filters.

\section{Results}

The Results section firstly outlines and compares the computational model simulations using three different implementations of the Metacognitive (Mratio) model. The hierarchical version (HMeta-d) is convincingly shown to be the most reliable in recovering simulated values of Mratio. Simulation results also establish the recoverability of regression parameters using the extended RHMeta-d model compared to the standard HMeta-d model. We then present the results from the example empirical analyses proposed in individuals with asthma and healthy controls, to demonstrate how the model outputs can be interpreted in light of example hypotheses. Lastly, we ascertain the utility of the novel task algorithm using both simulated and empirical results.

\subsection{Metacognitive model simulations}

The simulation results firstly demonstrate that utilizing the hierarchical Bayesian HMeta-d model fit allows adequate recovery of group Mratio values (Fig. 2). This recovery is possible even using as few as 20 trials per subject, with slightly larger uncertainties (demonstrated by the width of the highest density intervals) than those obtained for 40 and 60 trials. It is instructive to compare this to the alternative estimation methods: while MLE is able to recover an average Mratio value that is indeed representative of the simulated value, the uncertainty around these estimates (demonstrated by the width of the confidence intervals) is large when using even 60 trials per subject. Moreover, the confidence interval around these MLE estimates incorrectly encompasses zero for all group Mratio values below 1 . The recovery of the group Mratio using the Bayesian single subject fit also has large uncertainties and shows shrinkage effects towards zero, recovering Mratio values below the simulated values across all trial numbers tested here.

The second set of simulations were designed to probe the recoverability of single-subject Mratio values, for possible use in analyses comparing individual metacognitive performance against an external variable. Using the original HMeta-d model, we demonstrated that a post-hoc regression on the single-subject values was unable to accurately recover a group regression parameter simulated from the range $\beta=$ [-0.5:0.5] even when using 60 trials, with all confidence intervals on the regression parameters encompassing zero (Fig. 3). This is unsurprising given that the hierarchical model naturally shrinks single-subject 

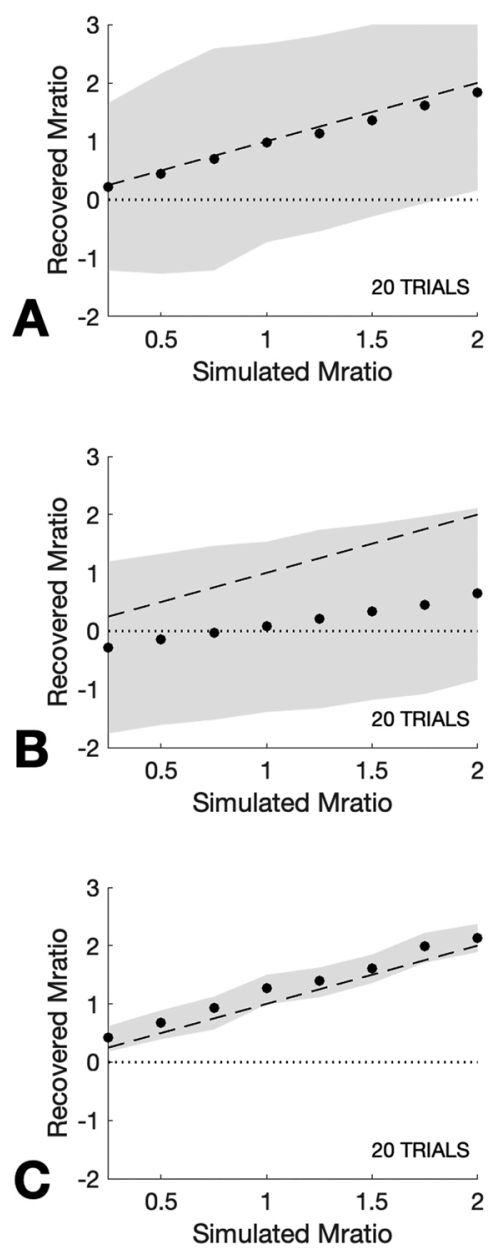

MLE FIT

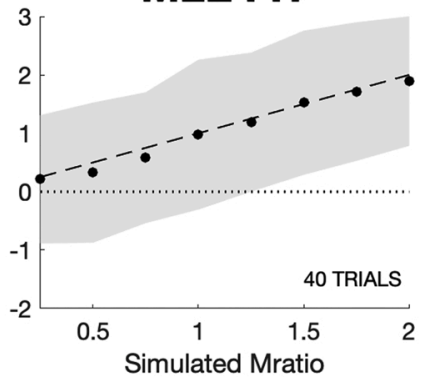

BAYES SINGLE FIT

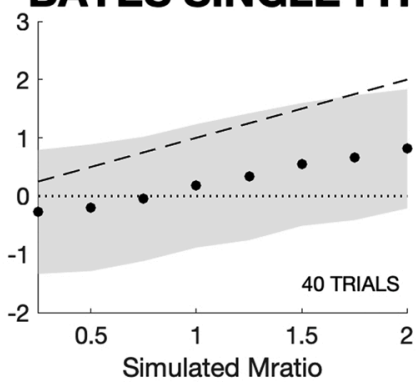

BAYES GROUP FIT

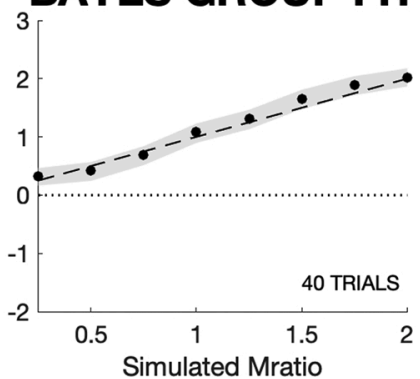

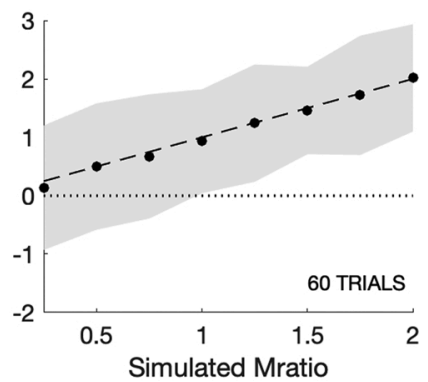
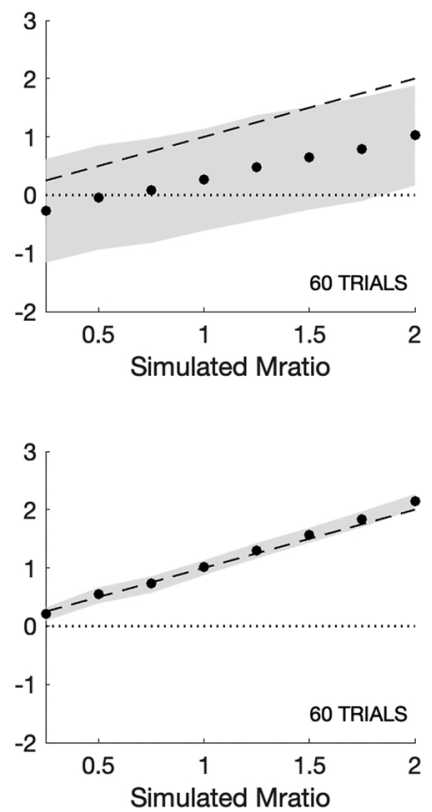

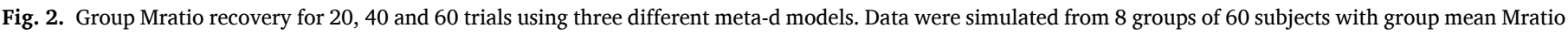

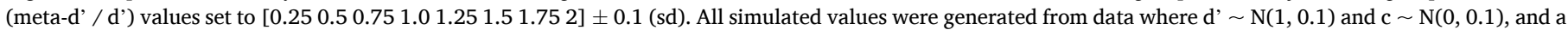

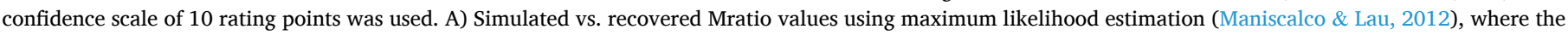

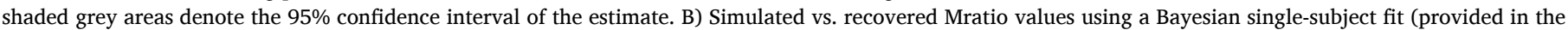

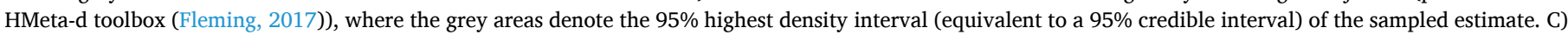

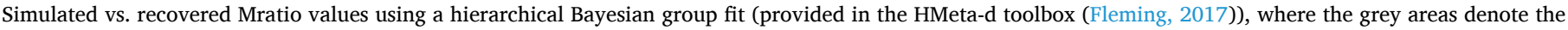

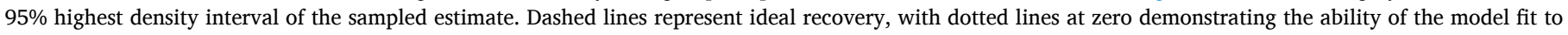
significantly recover group estimates (i.e. when confidence or highest density intervals do not include zero).
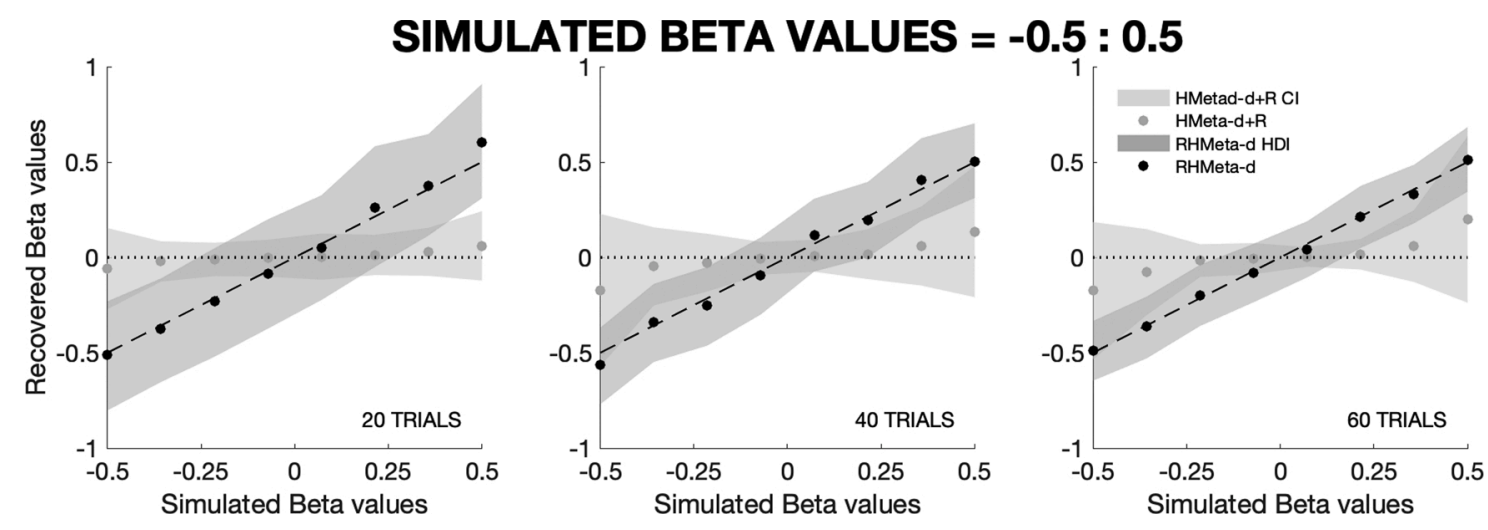

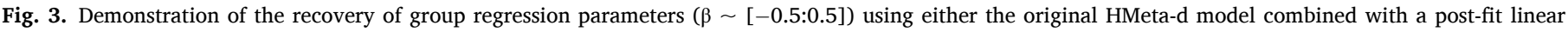

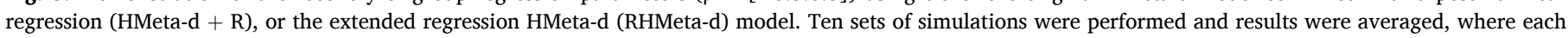

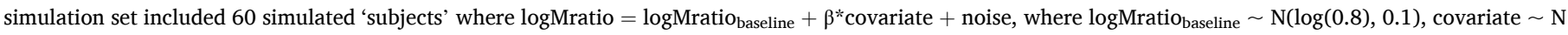

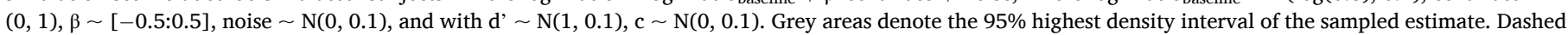

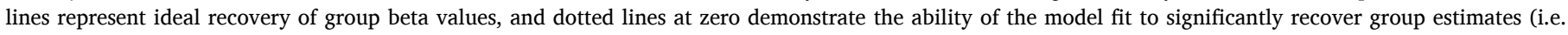
highest density intervals that do not including zero). 
estimates towards the group mean, losing information about individual differences. In contrast, the RHMeta-d model was able to significantly recover beta values of $\pm>0.2$ with 60 trials, $\pm>0.25$ with 40 trials, and $\pm>0.3$ with 20 trials (Fig. 3). The results for multiple regression models (with up to three covariates) at each of 20, 40 and 60 trials are presented in the Supplementary Material (Supplementary Fig. 4).

\subsection{Empirical data analyses}

When considering the comparisons between the asthma and control groups, no significant difference was found between the two groups for interoceptive sensitivity (number of filters $=$ mean \pm se: controls $=2.87$ \pm 0.29 , asthma $=2.80 \pm 0.20)$, decision bias $(\mathrm{c}=$ mean \pm se: controls $=$ $0.01 \pm 0.07$, asthma $=-0.01 \pm 0.06$ ), metacognitive bias (average confidence $\%=$ mean \pm se: controls $=66.57 \pm 2.99$, asthma $=69.74 \pm$ 2.13), nor metacognitive performance (Mratio $=$ mean \pm se: controls $=$ $0.83 \pm 0.14$, asthma $=0.79 \pm 0.12$ ) (Fig. 4). We then considered the relationship between breathing symptoms and metacognition in asthma only. While the data demonstrated a tendency for reduced metacognitive performance with higher symptom loads using a hierarchical regression analysis (RHMeta-d; Fig. 5), this was not statistically significant (determined by an HDI that does not encompass zero). Using a hierarchical regression approach, the beta parameter mean was estimated as $-0.22 \pm 0.16$ (se), with the beta HDI in the range $[-0.67$, 0.27] (Fig. 5).

\subsection{Task performance algorithm: Simulations and empirical data}

The results from both simulated and empirical data demonstrate the ability of the task algorithm to target performance towards a perceptual threshold that lies above chance (50\%) and below ceiling (100\%) performance (Fig. 6). Simulations conducted using the task algorithm (with a 'constant' staircase formulation) produced task accuracy scores with a mean of $74.1 \pm 8.7 \%$ (sd) and an accurate recovery of the $75 \%$ filter number, irrespective of the starting filter value (Fig. 6A). Empirical data collected using the Yes/No formulation of the task (with a constant staircase) produced a task accuracy with a mean of $68.9 \pm 7.4 \%$ (sd), with the threshold filter number spread between 1 and 8 filters (Fig. 6B). Both the simulations and real data demonstrate a feasible number of trials $(70.4 \pm 10.3$ (sd) trials for simulated results with 60 threshold trials, $69.6 \pm 10.0$ (sd) trials for empirical data) required to complete 60 trials at the threshold filter. In real terms, this indicates that it is possible to reliably measure respiratory interoception and metacognition in approximately one hour or less (assuming approximately 45-60 seconds to complete each trial). These estimates include a constraint whereby the algorithm was additionally programmed to continue until 30 trials are completed at the threshold filter number, followed by the option for manual intervention to instigate filter changes every ten trials if the accuracy moves out of acceptable bounds (experimenter decision required, depending on time taken and participant).

When utilizing a 'roving' staircase experimental design, simulations of the task algorithm produced task accuracy scores with a mean of 75.4 $\pm 8.0 \%$ (sd) and an accurate recovery of the $75 \%$ filter number, irrespective of the starting filter value (Supplementary Fig. 7A). When the first 60 trials from the constant staircase empirical data discussed above were analyzed as a roving staircase (i.e. the first 60 trials analyzed, regardless of filter intensity), the mean task accuracy was slightly reduced from $68.9 \%$ to $67.7 \%$, and the variance of the scores increased from $7.4 \%$ to $8.5 \%$ (sd) (Supplementary Fig. 7B). A final dataset, collected with explicit use of the roving staircase paradigm, demonstrated a mean accuracy of $69.7 \pm 11.7 \%$ (sd), and threshold filter numbers that ranged from 1 to 8 filters (Supplementary Fig. 7C). No additional trials are required when utilizing a roving staircase design, as all trials following the calibration step are included in the analysis. The
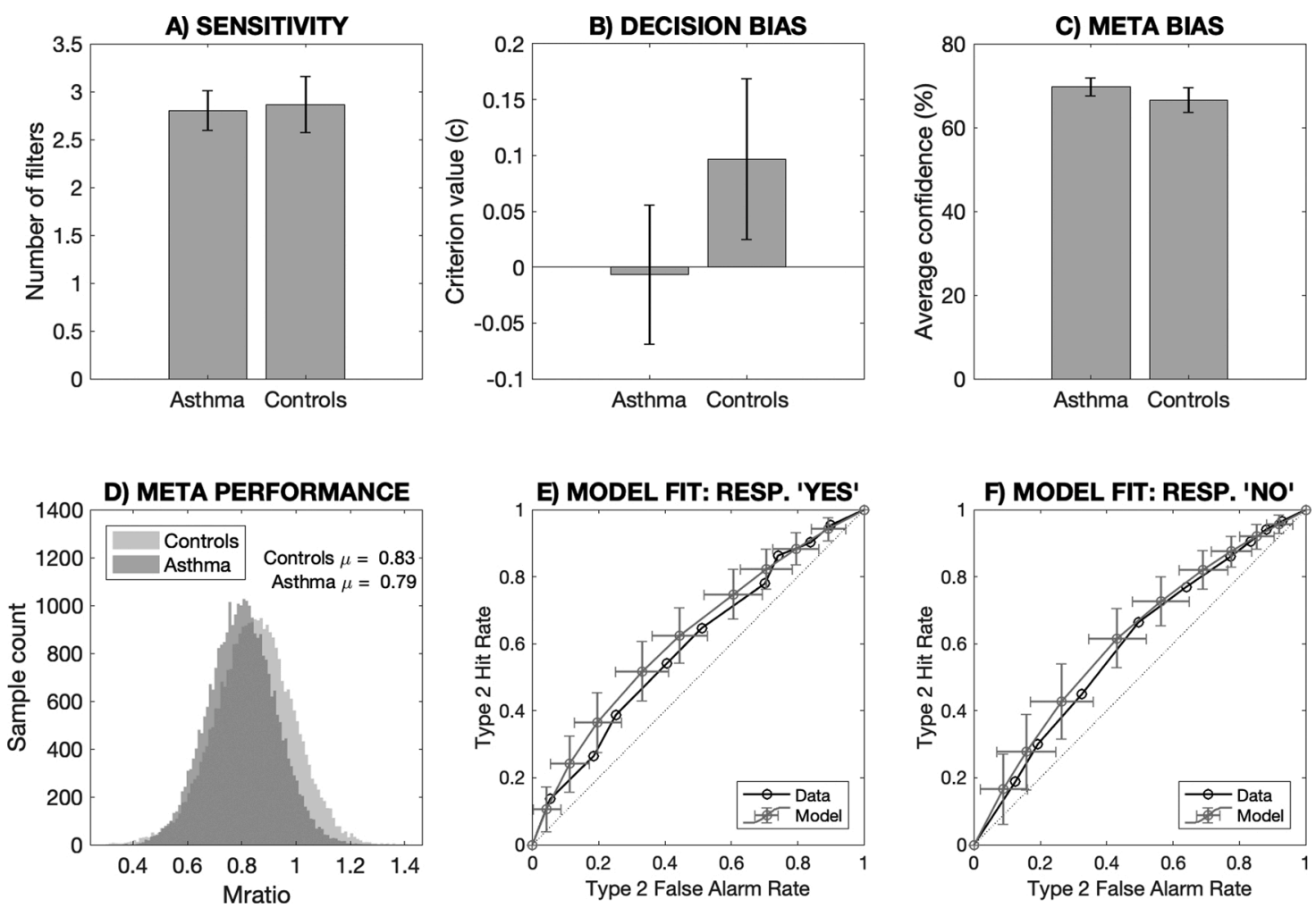

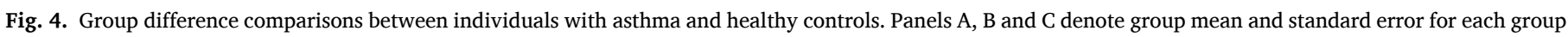

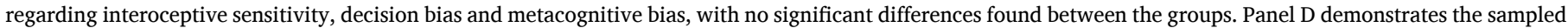

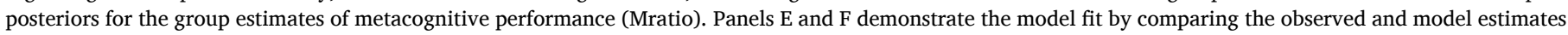

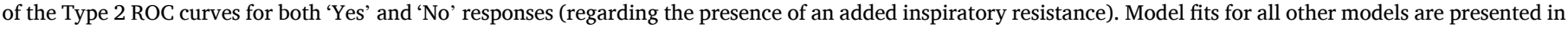
the Supplementary Material (Supplementary Fig. 5). 

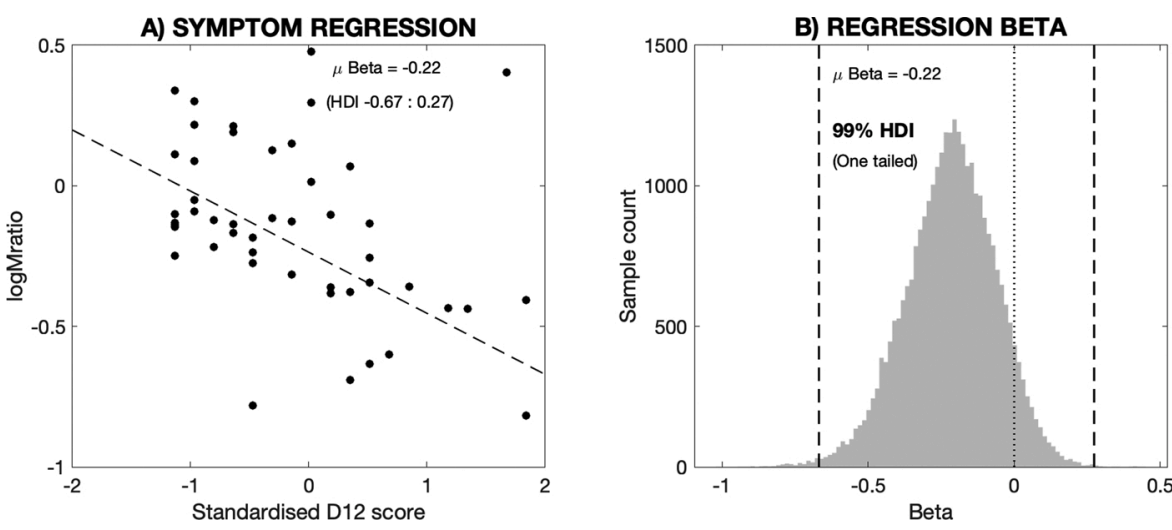

Fig. 5. Comparisons between metacognitive performance (logMratio) and symptom load in asthma, using a hierarchical regression analysis. A) A hierarchical regression predicting logMratio from the standardised D12 scores within asthma participants. The regression was fit using an extension of the HMeta-d model (RHMeta-d) in which the beta regression coefficient was fit simultaneously together with the logMratio scores. Dashed line represents the regression line from the model fit. B) The distribution of samples over the regression beta parameter (from panel A) fit using the RHMeta-d model. Dashed lines represent the two-tailed 99\% HDI which encompasses zero (dotted line) consistent with no significant relationship between D12 score and metacognitive efficiency in this dataset.
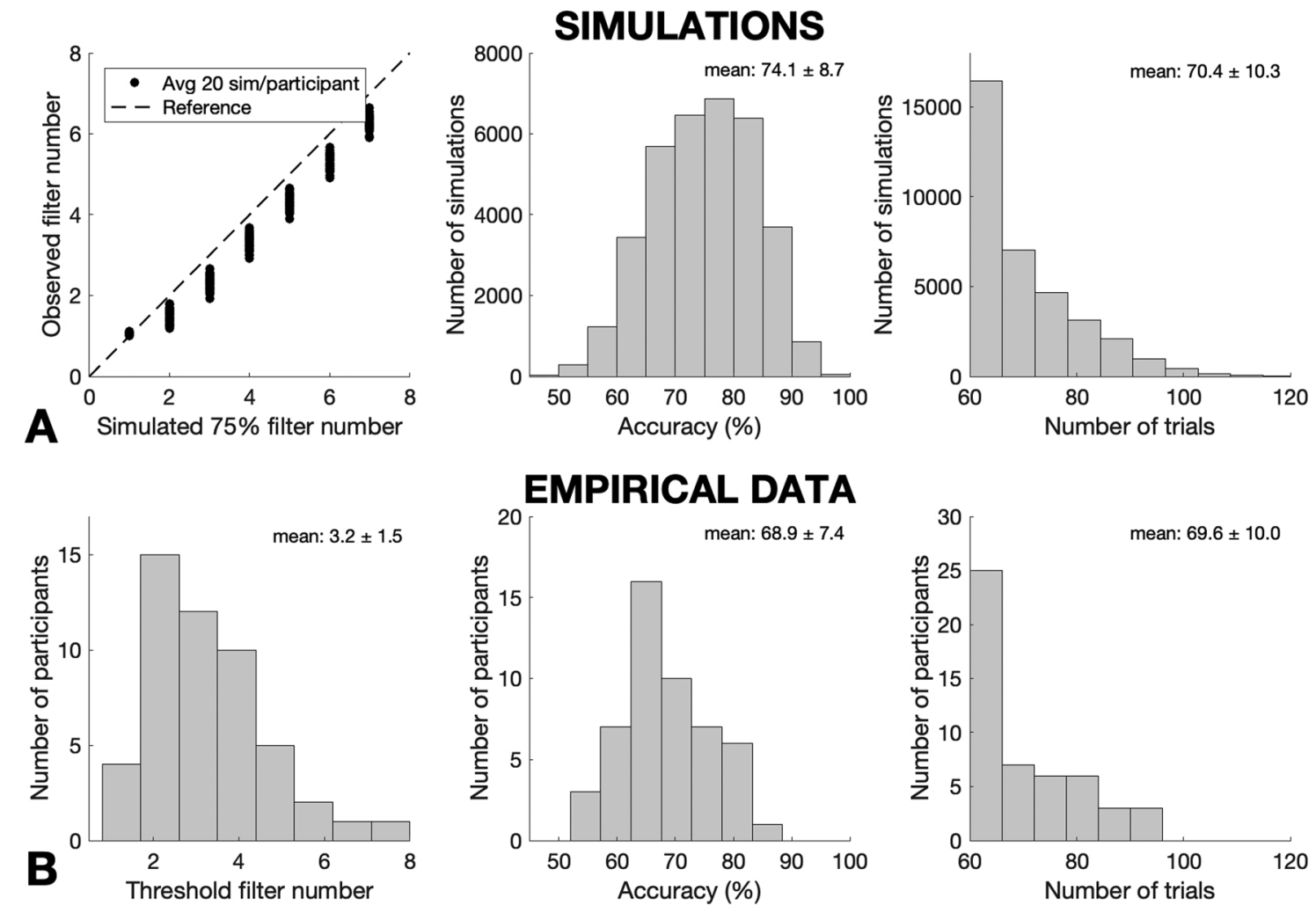

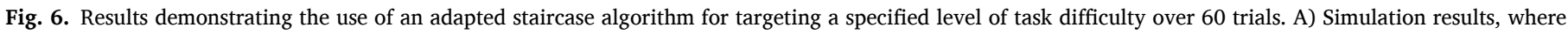

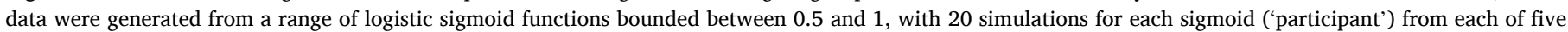

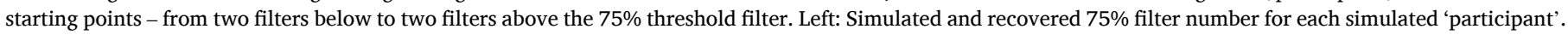

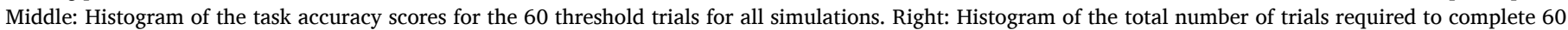

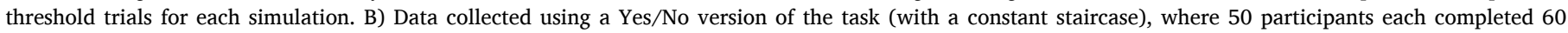

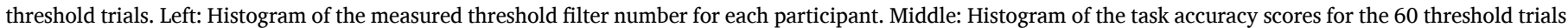

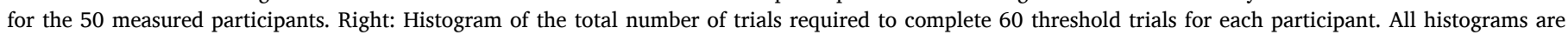
reported with mean \pm standard deviation.

simulated and empirical results from the calibration algorithm are presented in Supplementary Fig. 8.

Direct comparisons between the empirical data collected using the three methods (i.e. manual accuracy calculations every 10 trials (asthma dataset), the constant staircase design and the roving staircase design) are provided in Fig. 7. Data collected using a manual accuracy calibration (asthma and controls) produced task accuracy scores with a mean of $66.4 \pm 8.2 \%$ (sd), with a large number of additional trials (percentage of the number of threshold trials collected $=71.9 \pm 17.7 \%(\mathrm{sd})$ ) and two participants whose task accuracy was $\leq \mathbf{5 0} \%$. No difference in overall accuracy was found (Wilcoxon rank-sum tests) between any of the task designs (all $\mathrm{p}>0.05$ ), however the roving task design produced the largest standard deviation in the task accuracy across the methods (observed in Fig. 7). Additionally, while a significant number of additional trials were required when using the manual and constant staircase methods (Wilcoxon signed-rank tests, $\mathrm{p}<0.001$ for both tests against zero), the constant staircase significantly reduced the additional number of trials required from the manual method, both as an absolute number of trials and as a percentage of the number of threshold trials collected (Wilcoxon rank-sum tests, $\mathrm{p}<0.001$ for both tests).

Finally, while using a roving staircase design removes the possibility that any additional (non-analyzed) trials will be required, a tighter 


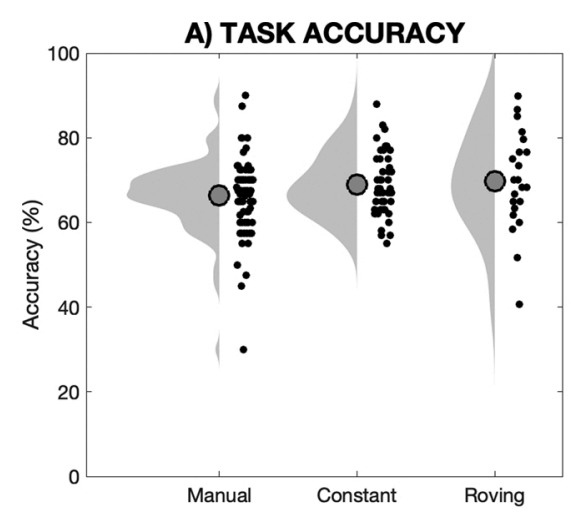

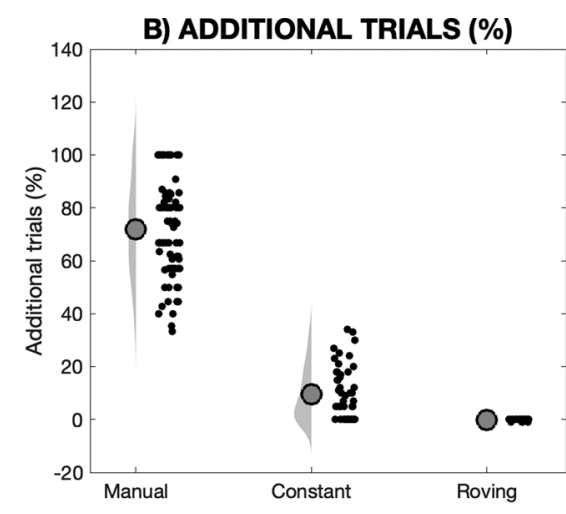

Fig. 7. Comparison of the three empirical datasets collected using different methods: 'Manual' = Manual staircase adjustment of filters via accuracy calculations every 10 trials; 'Constant' = Constant formulation of the staircase, where all analysed trials are collected at the same filter number; and 'Roving' = Roving staircase, where all trials (across different filter numbers) are used for data analysis. A) Comparison of task accuracy across the data collection methods, where no difference in accuracy was observed between any of the methods (all p > 0.05). B) Comparison of the additional trials required during data collection as percentage of the analysed threshold trials (N.B., no extra trials are collected for the roving staircase design). In Panel B, both the manual and constant staircase methods produced a significant percentage of additional trials (above zero, both $\mathrm{p}<0.001$ ), and the constant staircase significantly reduced the number / percentage of these

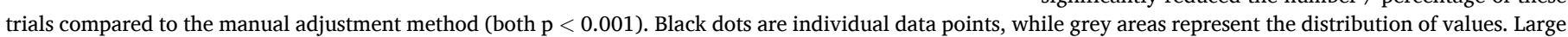
circles denote the group mean in each condition.

target accuracy band could be employed to better control the variance in performance accuracy (observed in Fig. 7), and the algorithm can be programmed to continue throughout all trials to better control task performance (instead of stopping at 30 trials, as occurs with the constant staircase). Therefore, we re-ran the task simulations using a difference in beta cumulative distribution functions between $75-70 \%$ (reduced from 80-65\%), with a lower bound on the acceptable probabilities increased from $20 \%$ to $30 \%$. The results of these simulations compared to the original thresholds can be seen in Supplementary Fig. 9, and these changes reduced the simulated standard deviation in task performance accuracy from 8.0 to 6.7 .

\section{Discussion}

\subsection{Main findings}

In this manuscript we demonstrate the utility of pairing a breathing perception task - the 'Filter detection task' - with the HMeta-d model of metacognition, to quantify the interoceptive domains of sensitivity, decision bias, metacognitive bias and metacognitive performance. Using simulations, we have shown how the use of a hierarchical model formulation (HMeta-d (Fleming, 2017)) can help overcome the challenge of low trial numbers when calculating metacognitive performance metrics using frameworks such as the meta-d model (Maniscalco \& Lau, 2012). We demonstrate how this hierarchical model can be extended to include a simultaneous hierarchical estimation of regression parameters linking metacognitive performance to individual difference variables. We also demonstrate the use of the model and appropriate statistics to answer research questions in an empirical dataset of healthy controls and individuals with asthma. However, we did not observe any group differences in our FDT measures (interoceptive sensitivity, interoceptive bias, metacognitive bias or metacognitive performance) within our empirical dataset (including usable data from 56 individuals with asthma and 30 healthy controls), who each completed $\sim 40$ trials (mean $\pm s d=42 \pm 10$ ) at their perceptual threshold using a manual staircase procedure. Whilst there may be no differences that exist between these groups in the measures tested, the noise associated with using only 40 trials when quantifying metacognition may also mask any underlying differences. Therefore, lastly, we introduce a task algorithm to help target performance accuracy towards $70-75 \%$ correct. This accuracy band is optimal for metacognitive analysis as it provides sufficient errors for analysis of confidence-accuracy relationships, while maintaining above-chance performance and accounting for any remaining variability in performance accuracy between participants. We demonstrate the effectiveness of this algorithm using both simulations and via empirical data comparisons when using either manual adjustment strategies or the staircase options provided by the toolbox (constant or roving staircase options).

\subsection{Computational models of breathing-related interoception and metacognition}

As interest in interoception-related research grows across neuroscience, psychiatry, physiology and other scientific communities, the importance of developing robust methodologies for quantification of interoceptive dimensions is paramount. While discussions regarding the validity of tasks such as heartbeat counting in the cardiac domain highlight the need for robust measures of interoception (Corneille, Desmedt, Zamariola, Luminet, \& Maurage, 2020), the FDT offers one route to overcoming some of these issues within the domain of respiration. Here, we highlight the feasibility of applying signal detection theory-derived computational models of both task and metacognitive performance, first introduced by Maniscalco and Lau (2012) (the meta-d model) and derived from theories of 'Type 2' performance (distinguishing between one's own correct and incorrect decisions (Galvin, Podd, Drga, \& Whitmore, 2003)). Utilizing these signal detection theory models firstly allows us to separate interoceptive sensitivity from decision biases within task (or 'Type 1') performance, both of which may be highly informative in disentangling drivers of altered interoception and have been previously quantified using inspiratory loading tasks in controls (Harver, Katkin, \& Bloch, 1993; Narbed, Marcer, \& Howell, 1982; Narbed, Marcer, Howell, \& Spencer, 1983) and children with asthma (Harver et al., 2013). Additionally, while there have been reports of possible blunted sensitivity to inspiratory resistive loads with anxiety prevalence of reporting medically unexplained symptoms with anxiety (Steinbrecher et al., 2011), and even early evidence for a potential relationship between symptom over-report and reduced interoceptive accuracy in healthy individuals (Bogaerts et al., 2008). Therefore, as a criterion shift may manifest as differences in interoceptive sensitivity, it is imperative to separate these measures both in healthy individuals and within clinical populations.

While perceptual sensitivity and bias metrics can be directly calculated from behavioural data, the estimation of metacognitive parameters such as meta-d' often require optimizing a model's predicted responses to match those observed within the data (Maniscalco \& Lau, 2012). However, here we demonstrate that the original meta-d model formulation (using maximum likelihood parameter estimates) is not able to significantly recover group Mratio values below 1, nor reliable estimates of individual subject scores when using the low number of trials that are practically feasible within interoceptive experiments (Fig. 2). Due to these constraints, here we instead explore the utility of hierarchical disorders (Tiller, Pain, \& Biddle, 1987), there is also an established 
formulations of the meta-d model (HMeta-d) derived by Fleming (2017), which can achieve good recovery of metacognitive performance parameters (e.g. Mratio) using as few as 20 trials per subject (Fig. 2). Importantly, the meta-d model allows us to differentiate relative metacognitive performance (i.e. metacognitive efficiency controlling for task performance) from absolute measures of metacognition, such as that calculated from the area under a type 2 ROC curve (Garfinkel et al., 2015, 2016a, 2016b). This is important because it is well-established that absolute measures of metacognition may be biased by differences in underlying task performance between individuals or conditions (Maniscalco \& Lau, 2012).

Beyond estimating group metrics of metacognition, often it may be desirable to estimate the relationship between individual metacognitive performance and an external measure of interest, for example a clinical score or other behavioural variable. While post-hoc regressions on single-subject parameter estimates are possible, hierarchical models tend to shrink single-subject estimates towards the group mean, thus losing information regarding individual differences and reducing the power of these types of analyses. To this end, we have developed and tested a hierarchical regression model, whereby multiple regression parameters can be simultaneously fit alongside the group logMratio within the HMeta-d model (RHMeta-d). We find that the sensitivity of the regression model in being able to accurately recover simulated beta coefficients is greatly enhanced when increasing from 20 to 40 and 60 trials, with the width of the posterior (represented by the HDI) notably reducing when trial number is increased (Fig. 3). We have also demonstrated the use of this regression approach in an empirical dataset in which interoceptive metacognitive performance was compared against breathlessness symptom reports (measured via the D12 questionnaire) in individuals with asthma (Fig. 4).

\subsection{FDT toolbox}

To aid the use of computational models within interoceptive experiments, we have developed a toolbox to run the FDT according to an accuracy-targeted performance algorithm (freely available for download: https://github.com/ofaull/FDT). While practicalities regarding the fixed step-size of each of the inspiratory resistance filters prevents us from utilizing established psychophysics staircases, we have instead developed an adapted staircase protocol which prompts adjustment of the filter load once the probability falls too far beyond our desired range of $70-75 \%$. As task performance control is carried out online at every trial, any variations in breathing physiology that may alter performance are dynamically accounted for across the task. Both simulations and empirical data show that this algorithm produces performances within the desired range required for employing the computational models described above, where participants need to be performing above chance but below $100 \%$ accuracy.

The demonstration of the FDT in the current manuscript utilized a Yes/No task formulation, where a participant is required to answer whether or not a resistance was added to the system ('Yes') or stayed the same ('No'). However, the toolbox also provides the option to employ a two-interval forced choice (2IFC) alternative if desired. While criticisms exist of the application of equal-variance signal detection theory metrics in Yes/No tasks (discussed previously (Peters et al., 2016)), we also see potential practical utility in using these task variants, for example to quantify measures akin to symptom over- or under-report by estimating the criterion parameter. However, if the metrics calculated from the FDT are to be compared with other perceptual tasks that are run as a 2-interval/alternative forced choice, then the 2IFC option may be desirable, allowing for comparable model assumptions across tasks.

Lastly, the toolbox also offers two alternative staircase options to control task performance in both the Yes/No or 2IFC formulation. In the original protocol presented by Garfinkel and colleagues (Garfinkel et al., 2016a), the aim was to collect 20 usable trials at a specific number of filters where performance first fell below $75 \%$, thus corresponding to the participant's perceptual threshold and providing a measure of interoceptive sensitivity. Whilst the number of additional (unused) trials required can be greatly reduced from using a standard staircase procedure by employing the adapted staircase algorithm in the toolbox (Fig. 7), an alternative approach is to employ a 'roving' staircase, whereby all trials are used in the calculation of interoceptive measures, and interoceptive sensitivity is taken as an average of the filter numbers used across trials. As the risk of needing additional trials is removed, this approach allows experimenters to tighten accuracy thresholds to improve task performance control, as the aim of this staircase is no longer to find a single filter that elicits the desired accuracy. This roving staircase option would likely prove a more viable alternative if using the FDT in a clinical setting, removing the possibility of any additional trials while maintaining adequate representations of interoceptive sensitivity. We note however that roving staircases also have potential downsides in artificially inflating estimates of metacognitive sensitivity when compared to constant-stimulus designs (see Rahnev and Fleming (2019) for further discussion of this issue).

\subsection{Limitations}

While this experimental setup provides a progression towards measuring quantities related to interoception of breathing, a number of limitations exist that could be addressed in future work. The first of these is that while the resistance applied is static, the resulting pressure differential across the resistance is flow-dependent, such that larger inspiratory flow will generate larger inspiratory pressure differences (see Supplementary Material for further details). Furthermore, inherent resting resistance and inspiratory pressures are also variable between individuals, depending on factors such as anatomical structure of the airways and physiological differences in inspiratory musculature. Therefore, if measures of inspiratory pressure and flow were added to the system, more detailed quantification of perceptual sensitivity may be determined by considering the changes in both the inspiratory pressure and flow (relative to the baseline breaths) that were required to detect the resistance. The use of mouth pressure, in particular, could be used as a replacement for the number of filters as a more nuanced measure of interoceptive sensitivity. As pressure will change in response to both changes in resistance as well as inspiratory flow (see Supplementary Fig. 3 for details), this measure would thus incorporate any inter-filter and inter-participant inspiratory flow variability. Importantly, differences in ventilatory properties between individuals with asthma and healthy controls may have contributed to the null finding where no difference in perceptual sensitivity was observed between the groups, despite previous reports of altered perception in asthma (Boulay \& Boulet, 2013; Janssens et al., 2009; Kendrick et al., 1993; Teeter \& Bleecker, 1998). The downside of these additional measures would be the loss of some of the task simplicity, and thus its feasibility for use in a wide range of settings.

Whilst adding physiological measures would improve the accuracy of the perceptual sensitivity measures, it is worth noting that the addition of these physiological measures would not likely alter the metacognitive performance scores. This is due to two factors: the task both controlling performance and the meta-d model accounting for any remaining performance variability (seen in Fig. 7); this renders the metacognitive performance scores independent of interoceptive sensitivity (Fleming \& Lau, 2014). Therefore, the null effect observed in the metacognitive performance domain between individuals with asthma and healthy controls may reflect no observable difference between the groups as a whole. Alternatively, it is possible that "interoceptive" sub-types or phenotype of asthma exist, whereby some individuals with asthma experience altered metacognition compared to healthy controls, whilst others do not.

A further notable limitation of the current version of the FDT is the time required for completion of the task. The original manual staircase proposed by Garfinkel and colleagues (Garfinkel et al., 2016a) (used in 
the collection of the asthma data presented here) could require more than $60 \mathrm{~min}$ to acquire the final 20 trials used in the analysis, and other inspiratory load paradigms measuring only objective performance also require up to $60 \mathrm{~min}$ to collect only 7 trials at each level of resistance (Davenport et al., 2007). Using this manual staircase and $\sim 40$ trials in each participant, we were unable to identify any differences in interoceptive sensitivity, interoceptive bias, metacognitive bias or metacognitive performance between individuals with asthma and healthy controls. Additionally, while worsened D12 scores appeared to relate to reduced metacognitive performance in asthma (Fig. 5), this relationship did not reach statistical significance. To aid the collection of more trials in order to reduce the noise associated with each of our measures, the implementation of the FDT staircase allows for up to 60 usable trials in approximately the same amount of time $(45-70 \mathrm{~min})$. To further improve the ecological validity of the task, a roving staircase design with only 40 trials could be employed, and a reduction in the number of breaths per interval (currently 3 breaths) could be considered; these would dramatically reduce the time required for task completion to approximately $20-30 \mathrm{~min}$ per participant. However, these options have not yet been tested in practice.

An additional limitation of the current design is the currently linear staircase step-sizes induced by adding or removing a filter, and their lack of highly-accurate factory calibrations. While the resistance increments of $0.42 \mathrm{~cm} \mathrm{H}_{2} \mathrm{O} / \mathrm{L} \cdot \mathrm{sec}^{-1}$ provided here (see Supplementary Material for details) are similar to those used in previous paradigms such as that by

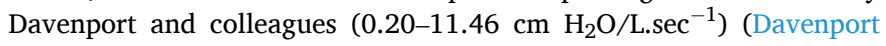
et al., 2007), sophisticated electronic devices that can deliver very variable small resistances $\left(<0.5 \mathrm{~cm} \mathrm{H}_{2} \mathrm{O} / \mathrm{L}_{\mathrm{sec}} \mathrm{Se}^{-1}\right)$ are not yet widely available. The development of such a device could allow for variable step-sizes, reduce the time required to manually change filters, and allow greater control over inspiratory flow rate and volume to reduce breath-by-breath variability. Such devices may either incrementally change resistance using techniques such as an adjustable aperture, or with even further sophistication establish a constant inspiratory pressure via biofeedback devices. The possible improvements in control over the staircase step-size may also allow for more established staircase procedures (such as QUEST) to be implemented here, where the fine-grain coverage of stimulus strengths required to accurately identify perceptual threshold may then become available. Furthermore, automated developments would also greatly reduce the current experimenter burden of having to manually change filters and interact with the task algorithm at every trial.

While the limitations in the current measures of perceptual sensitivity are worth observing and improving, these limitations do not discount the utility of the current measures. While the noise of the perceptual sensitivity metrics will be notable (but not necessarily insurmountable), the control of task performance allows the metacognitive measures to be somewhat independent of this noise. Furthermore, the measure of metacognitive performance directly accounts for any remaining differences in task performance by creating a ratio of meta-d' / d'. Finally, keeping participants comfortable and reinforcing the notion that the task should be performed with normal pace and depth of breathing should limit large differences in inspiratory flow and pressure, and filters could be additionally numbered to ensure consistency in incremental steps.

\section{Conclusions}

Here we present a breathing-related interoceptive application of a computational model designed to tease apart important aspects of perception: sensitivity, decision bias, metacognitive bias and metacognitive performance. Whilst interoceptive experiments often suffer from low trial numbers, by combining a breathing perception task with a hierarchical statistical model we were able to develop a robust algorithm to control task performance while maximizing the number of useful trials for analysis.
The FDT toolbox is freely available for download (https://github.co $\mathrm{m} /$ ofaull/FDT), as are the statistical methods employed (MLE model: htt p://www.columbia.edu/-bsm2105/type2sdt/; HMeta-d and RHMeta-d: https://github.com/metacoglab/HMeta-d/).

\section{Declaration of Competing Interest}

The authors have no conflicts of interest to disclose.

\section{Acknowledgements}

Data provided in this manuscript is not yet publicly available: Please contact authors for data access. OKH (née Faull) was supported as a Marie Skłodowska-Curie Postdoctoral Fellow from the European Union's Horizon 2020 research and innovation programme (under the Grant Agreement No 793580), and as a Rutherford Discovery Fellow from the Royal Society of New Zealand. MA is supported by a Lundbeckfonden Fellowship from the Lundbeckfonden Foundation (R272-2017-4345), and an AIAS-COFUND II fellowship, which is supported by the Marie Skłodowska-Curie actions under the European Union's Horizon 2020 (Grant agreement no 754513), and the Aarhus University Research Foundation. SJH was supported by the grant \#2017-403 of the Postdoctoral Fellowshi from the Strategic Focal Area "Personalized Health and Related Technologies (PHRT)" of the ETH Domain. KES is supported by the René and Susanne Braginsky Foundation and the University of Zurich. KTSP was supported by the JABBS Foundation for this work. SMF is supported by a Sir Henry Dale Fellowship jointly funded by the Wellcome Trust and Royal Society (206648/Z/17/Z).

\section{Appendix A. Supplementary data}

Supplementary material related to this article can be found, in the online version, at doi:https://doi.org/10.1016/j.biopsycho.2021.10 8185.

\section{References}

Allen, M. (2020). Unravelling the neurobiology of interoceptive inference. Trends in Cognitive Sciences, 24, 265-266.

Bocanegra, B. R., \& Zeelenberg, R. (2009). Emotion improves and impairs early vision. Psychological Science, 20, 707-713.

Bogaerts, K., Notebaert, K., Van Diest, I., Devriese, S., De Peuter, S., \& Van den Bergh, O. (2005). Accuracy of respiratory symptom perception in different affective contexts. Journal of Psychosomatic Research, 58, 537-543.

Bogaerts, K., Millen, A., Li, W., De Peuter, S., Van Diest, I., Vlemincx, E., et al. (2008). High symptom reporters are less interoceptively accurate in a symptom-related context. Journal of Psychosomatic Research, 65, 417-424.

Boulay, M.-E., \& Boulet, L.-P. (2013). Discordance between asthma control clinical, physiological and inflammatory parameters in mild asthma. Respiratory Medicine, 107, 511-518.

Brefczynski, J. A., \& DeYoe, E. A. (1999). A physiological correlate of the "spotlight" of visual attention. Nature Neuroscience, 2, 370-374.

Chou, Y. L., \& Davenport, P. W. (2007). The effect of increased background resistance on the resistive load threshold for eliciting the respiratory-related evoked potential. Journal of Applied Physiology, 103, 2012-2017.

Cooper, C. L., Parry, G. D., Saul, C., Morice, A. H., Hutchcroft, B. J., Moore, J., et al. (2007). Anxiety and panic fear in adults with asthma: Prevalence in primary care BMC Family Practice, 8, 1-7.

Corneille, O., Desmedt, O., Zamariola, G., Luminet, O., \& Maurage, P. (2020). A heartfelt response to Zimprich et al. (2020), and Ainley et al. (2020)'s commentaries Acknowledging issues with the HCT would benefit interoception research. Biological Psychology, 152, Article 107869.

Daubenmier, J., Sze, J., Kerr, C. E., Kemeny, M. E., \& Mehling, W. (2013). Follow your breath: Respiratory interoceptive accuracy in experienced meditators. Psychophysiology, 50, 777-789.

Davenport, P. W., Chan, P.-Y. S., Zhang, W., \& Chou, Y.-L. (2007). Detection threshold for inspiratory resistive loads and respiratory-related evoked potentials. Journal of Applied Physiology, 102, 276-285.

De Peuter, S., Lemaigre, V., Van Diest, I., \& Van den Bergh, O. (2008). Illness-specific catastrophic thinking and overperception in asthma. Health Psychology, 27, 93-99.

De Peuter, S., Van Diest, I., Lemaigre, V., Verleden, G., Demedts, M., \& Van den Bergh, O. (2004). Dyspnea: The role of psychological processes. Clinical Psychology Review, 24, $557-581$. 
Fleming, S. M. (2017). HMeta-d: hierarchical Bayesian estimation of metacognitive efficiency from confidence ratings. Neuroscience of Consciousness. https://doi.org/ 10.1093/nc/nix007, 377-14.

Fleming, S. M., \& Lau, H. C. (2014). How to measure metacognition. Frontiers in Human Neuroscience, 8, 443.

Gallego, J., Nsegbe, E., \& Durand, E. (2001). Learning in respiratory control. Behavior Modification, 25, 495-512.

Galvin, S. J., Podd, J. V., Drga, V., \& Whitmore, J. (2003). Type 2 tasks in the theory of signal detectability: Discrimination between correct and incorrect decisions. Psychonomic Bulletin \& Review, 10, 843-876.

Garfinkel, S. N., Seth, A. K., Barrett, A. B., Suzuki, K., \& Critchley, H. D. (2015). Knowing your own heart: Distinguishing interoceptive accuracy from interoceptive awareness. Biological Psychology, 104, 65-74.

Garfinkel, S. N., Manassei, M. F., Hamilton-Fletcher, G., In den Bosch, Y., Critchely, H. D., \& Engels, M. (2016a). Interoceptive dimensions across cardiac and respiratory axes. Philosophical Transactions of the Royal Society of London Series B, Biological Sciences, 371, 20160014-10.

Garfinkel, S. N., Tiley, C., O-Keeffe, S., Harrison, N. A., Seth, A. K., \& Critchley, H. D. (2016b). Discrepancies between dimensions of interoception in autism: Implications for emotion and anxiety. Biological Psychology, 114, 117-126.

Gelman, A., Carlin, J. B., Stern, H. S., Dunson, D. B., Vehtari, A., \& Rubin, D. B. (2020), Models for robust inference (pp. 435-446). CRC Press.

Green, D. M., \& Swets, J. A. (1966). Signal detection theory and psychophysics.

Harver, A., Katkin, E. S., \& Bloch, E. (1993). Signal-detection outcomes on heartbeat and respiratory resistance detection tasks in male and female subjects. Psychophysiology, 30, 223-230.

Harver, A., Kotses, H., Ersek, J., Humphries, C. T., Ashe, W. S., \& Black, H. R. (2013). Effects of feedback on the perception of inspiratory resistance in children with persistent asthma. Psychosomatic Medicine, 75, 729-736.

Isaac, M. L., \& Paauw, D. S. (2014). Medically unexplained symptoms. Medical Clinics of NA, 98, 663-672.

Janssens, T., De Peuter, S., Stans, L., Verleden, G., Troosters, T., Decramer, M., et al. (2011). Dyspnea perception in COPD: Association between anxiety, dyspnea-related fear, and dyspnea in a pulmonary rehabilitation program. CHEST Journal, 140, 618-625.

Janssens, T., Verleden, G., De Peuter, S., Van Diest, I., \& Van den Bergh, O. (2009). Inaccurate perception of asthma symptoms: A cognitive-affective framework and implications for asthma treatment. Clinical Psychology Review, 29, 317-327.

Johnson, B. D., Weisman, I. M., Zeballos, R. J., \& Beck, K. C. (1999). Emerging concepts in the evaluation of ventilatory limitation during exercise the exercise tidal flowvolume loop. Chest, 116, 488-503.

Katon, W. J., Richardson, L., Lozano, P., \& McCauley, E. (2004). The relationship of asthma and anxiety disorders. Psychosomatic Medicine, 66, 349.

Kendrick, A. H., Higgs, C. M., Whitfield, M. J., \& Laszlo, G. (1993). Accuracy of perception of severity of asthma: Patients treated in general practice. BMJ, 307, 422-424.

Khalsa, S. S., Adolphs, R., Cameron, O. G., Critchley, H. D., Davenport, P. W., Feinstein, J. S., et al. (2017). Interoception and mental health: A roadmap. Biological Psychiatry: Cognitive Neuroscience and Neuroimaging, 1-57. https://doi.org/10.1016/ j.bpsc. 2017.12.004

Khatib, M. F., Oku, Y., \& Bruce, E. N. (1991). Contribution of chemical feedback loops to breath-to-breath variability of tidal volume. Respiration Physiology, 83, 115-127.

Kingdom, F., \& Prins, N. (2016). Psychophysics: A practical introduction. Elsevier.

Koskela, H. O. (2007). Cold air-provoked respiratory symptoms: The mechanisms and management. International Journal of Circumpolar Health, 66, 91-100.

Koskela, H., \& Tukiainen, H. (1995). Facial cooling, but not nasal breathing of cold air, induces bronchoconstriction: A study in asthmatic and healthy subjects. The European Respiratory Journal, 8, 2088-2093.

Lee, A. L. F., Ruby, E., Giles, N., \& Lau, H. (2018). Cross-domain association in metacognitive efficiency depends on first-order task types. Frontiers in Psychology, 9 , 440-10.

Maniscalco, B., \& Lau, H. (2012). A signal detection theoretic approach for estimating metacognitive sensitivity from confidence ratings. Consciousness and Cognition, 21, 422-430.

Marlow, L. L., Faull, O. K., Finnegan, S. L., \& Pattinson, K. T. S. (2019). Breathlessness and the brain: The role of expectation. Current Opinion in Supportive and Palliative Care, 13, 200-210.

Moutoussis, M., Hopkins, A. K., \& Dolan, R. J. (2018). Hypotheses about the relationship of cognition with psychopathology should be tested by embedding them into empirical priors. Frontiers in Psychology, 9, 1-3.
Narbed, P. G., Marcer, D., \& Howell, J. B. L. (1982). The contribution of the accelerating phase of inspiratory flow to resistive load detection in man. Clinical Science, 62 , 367-372.

Narbed, P. G., Marcer, D., Howell, J. B. L., \& Spencer, E. (1983). A signal detection theory analysis of the effect of chest cage restriction upon the detection of inspiratory resistive loads. Clinical Science, 64, 417-421.

Nimnuan, C., Hotopf, M., \& Wessely, S. (2001). Medically unexplained symptoms an epidemiological study in seven specialities. Journal of Psychosomatic Research, 51, 361-367.

Owens, A. P., Allen, M., Ondobaka, S., \& Friston, K. J. (2018). Interoceptive inference: From computational neuroscience to clinic. Neuroscience and Biobehavioral Reviews, 90, 174-183.

Paulus, M. P. (2013). The breathing conundrum - Interoceptive sensitivity and anxiety. Depression and Anxiety, 30, 315-320.

Peters, M. A. K., Ro, T., \& Lau, H. (2016). Who's afraid of response bias? Neuroscience of Consciousness, 2016. niw001-8.

Petzschner, F. H., Weber, L. A. E., Gard, T., \& Stephan, K. E. (2017). Computational psychosomatics and computational psychiatry: Toward a joint framework for differential diagnosis. Biological Psychiatry, 1-10. https://doi.org/10.1016/j. biopsych.2017.05.012

Pezzulo, G., Rigoli, F., \& Friston, K. (2015). Active Inference, homeostatic regulation and adaptive behavioural control. Progress in Neurobiology, 134, 17-35.

Porges, S. W. (1993). Body perception questionnaire (pp. 1-3).

Put, C., Van den Bergh, O., Van Ongeval, E., De Peuter, S., Demedts, M., \& Verleden, G. (2004). Negative affectivity and the influence of suggestion on asthma symptoms. Journal of Psychosomatic Research, 57, 249-255.

Rahnev, D., \& Fleming, S. M. (2019). How experimental procedures influence estimates of metacognitive ability. Neuroscience of Consciousness, 2019, 415-419.

Richardson, L. P., Lozano, P., Russo, J., McCauley, E., Bush, T., \& Katon, W. (2006). Asthma symptom burden: Relationship to asthma severity and anxiety and depression symptoms. Pediatrics, 118, 1042-1051.

Rimington, L. D., Davies, D. H., Lowe, D., \& Pearson, M. G. (2001). Relationship between anxiety, depression, and morbidity in adult asthma patients. Thorax, 56, 266-271.

Rouault, M., Seow, T., Gillan, C. M., \& Fleming, S. M. (2018). Psychiatric symptom dimensions are associated with dissociable shifts in metacognition but not task performance. Biological Psychiatry, 1-9. https://doi.org/10.1016/j. biopsych.2017.12.017

Ruehland, W. R., Rochford, P. D., Trinder, J., Spong, J., \& O’Donoghue, F. J. (2019). Evidence against a subcortical gate preventing conscious detection of respiratory load stimuli. Respiratory Physiology \& Neurobiology, 259, 93-103.

Smoller, J. W., Pollack, M. H., Otto, M. W., Rosenbaum, J. F., \& Kradin, R. L. (1996). Panic anxiety, dyspnea, and respiratory disease: Theoretical and clinical considerations. American Journal of Respiratory and Critical Care Medicine, 154, 6-17.

Stanislaw, H., \& Todorov, N. (1999). Calculation of signal detection theory measures. Behavior Research Methods, Instruments, \& Computers: A Journal of the Psychonomic Society, Inc, 31, 137-149.

Steinbrecher, N., Koerber, S., Frieser, D., \& Hiller, W. (2011). The prevalence of medically unexplained symptoms in primary care. PSYM, 52, 263-271.

Stephan, K. E., Manjaly, Z. M., Mathys, C. D., Weber, L. A. E., Paliwal, S., Gard, T., et al. (2016). Allostatic self-efficacy: A metacognitive theory of dyshomeostasis-induced fatigue and depression. Frontiers in Human Neuroscience, 10, 49-27.

Summerfield, C., \& Egner, T. (2009). Expectation (and attention) in visual cognition. Trends in Cognitive Sciences, 13, 403-409.

Teeter, J. G., \& Bleecker, E. R. (1998). Relationship between airway obstruction and respiratory symptoms in adult asthmatics. Chest, 113, 272-277.

Tiller, J., Pain, M., \& Biddle, N. (1987). Anxiety disorder and perception of inspiratory resistive loads. Chest, 91, 547-551.

Van den Bergh, O., Winters, W., Devriese, S., Van Diest, I., Vos, G., \& De Peuter, S. (2004). Accuracy of respiratory symptom perception in persons with high and low negative affectivity. Psychology \& Health, 19, 213-222.

Van den Bergh, O., Witthöft, M., Petersen, S., \& Brown, R. J. (2017). Symptoms and the body: Taking the inferential leap. Neuroscience \& Biobehavioral Reviews, 74, 185-203.

Watson, A. B. (2017). QUEST+: A general multidimensional Bayesian adaptive psychometric method. Journal of Vision, 17, 10-10.

Watson, A. B., \& Pelli, D. G. (1983). QUEST: a Bayesian adaptive psychometric method. Perception \& Psychophysics, 33, 113-120.

Yorke, J., Moosavi, S. H., Shuldham, C., \& Jones, P. W. (2010). Quantification of dyspnoea using descriptors: Development and initial testing of the Dyspnoea- 12. Thorax, 65, 21-26. 